\title{
Efficacy and Safety of Vacuum-Assisted Excision for Benign Breast Mass Lesion: A Meta-Analysis
}

\author{
Hee-Seon Yoo ${ }^{1}$, Wu-Seong Kang ${ }^{2}{ }^{\mathbb{D}}$, Jung-Soo Pyo ${ }^{3, *(\mathbb{D}}$ and Junghan Yoon ${ }^{4, *}$ \\ 1 Department of Surgery, Mizpia Hospital, Gwangju-si 61963, Gyeonggi-do, Korea; zinbang38@naver.com \\ 2 Department of Trauma Surgery, Cheju Halla General Hospital, Jeju-si 63127, Jeju-do, Korea; \\ wuseongkang@naver.com \\ 3 Department of Pathology, Uijeongbu Eulji Medical Center, Eulji University School of Medicine, Dongil-ro, \\ Uijeongbu-si 11759, Gyeonggi-do, Korea \\ 4 Department of Surgery, Chonnam National University Hwasun Hospital, \\ Hwasun-eup 58128, Jeollanam-do, Korea \\ * Correspondence: anapyojs@gmail.com (J.-S.P.); jhyoon@jnu.ac.kr (J.Y.)
}

Citation: Yoo, H.-S.; Kang, W.-S.; Pyo, J.-S.; Yoon, J. Efficacy and Safety of Vacuum-Assisted Excision for Benign Breast Mass Lesion: A Meta-Analysis. Medicina 2021, 57, 1260. https://doi.org/10.3390/ medicina57111260

Academic Editor: Wisit

Cheungpasitporn

Received: 7 October 2021

Accepted: 16 November 2021

Published: 17 November 2021

Publisher's Note: MDPI stays neutral with regard to jurisdictional claims in published maps and institutional affiliations.

Copyright: (c) 2021 by the authors. Licensee MDPI, Basel, Switzerland. This article is an open access article distributed under the terms and conditions of the Creative Commons Attribution (CC BY) license (https:/ / creativecommons.org/licenses/by/ $4.0 /)$.
Abstract: Background and Objective: Breast mass lesions are common; however, determining the malignant potential of the lesion can be ambiguous. Recently, to evaluate breast mass lesions, vacuum-assisted excision (VAE) biopsy has been widely used for both diagnostic and therapeutic purposes. This study aimed to investigate the therapeutic role of VAE. Materials and Methods: Relevant articles were obtained by searching PubMed and EMBASE on 3 September 2021. Meta-analyses were performed using odds ratios and proportions. To assess heterogeneity, we conducted a subgroup analysis and meta-regression tests. Results: Finally, 26 studies comprising 18,170 patients were included. All of these were observational studies. The meta-analysis showed that the complete resection rate of VAE was 0.930 . In the meta-regression test, there was no significant difference. The meta-analysis showed a recurrence rate of 0.039 in the VAE group. The meta-regression test showed no statistical significance. Postoperative hematoma, pain, and ecchymosis after VAE were 0.092, 0.082 , and 0.075 , respectively. Conclusion: VAE for benign breast lesions showed favorable outcomes with respect to complete resection and complications. This meta-analysis suggested that VAE for low-risk benign breast lesions is a reasonable option for both diagnostic and therapeutic purposes.

Keywords: breast mass; vacuum assisted excision; meta-analysis; systematic review

\section{Introduction}

Breast mass lesions are very common, but it can be difficult to determine their malignant potential [1]. Because breast cancer is one of the leading causes of mortality worldwide, it is crucial to detect malignant lesions [1,2]. Although the performance of the imaging system to evaluate the potential of malignancy has increased, biopsy is the final informative method for diagnosis [3]. Histologic confirmation is enabled by fine-needle aspiration, core needle biopsy, open excision, and vacuum-assisted excision (VAE) biopsy. Among these modalities, fine-needle aspiration and core needle biopsy can only be used for diagnostic purposes, but it has some limitations [3]. Fine-needle aspiration and core needle biopsy cannot examine the whole mass, whereas VAE can perform a full examination of the entire mass. Owing to the advantage that VAE can remove the entire mass region, VAE has recently been widely used for both diagnostic and therapeutic purposes [3-5]. In 2002, the US Food and Drug Administration (FDA) approved VAE for the removal of benign lesions. Although VAE can remove a large amount of tissue, open surgical excision remains the gold standard for large palpable masses [6]. Thus, the indications and efficacy of VAE remain controversial. Although traditional open excision is the most effective method for removing all mass lesions, it results in scarring, which is not preferred by women. Most surgeons would respect this patient's preference if the procedure is secure. 
This study aimed to investigate the efficacy and safety of VAE. We investigated the complete resection rate, recurrence rate, and complications after VAE for benign breast mass lesions.

\section{Materials and Methods}

\subsection{Published Study Search and Selection Criteria}

This study was performed in accordance with the Preferred Reporting Items for Systematic Reviews and Meta-Analyses [7]. Relevant articles were obtained by searching PubMed and EMBASE on 3 September 2021. These databases were searched using the following keywords: “(breast) AND (benign) AND (vacuum-assisted OR mammotome) AND (excision)". The titles and abstracts of all searched articles were screened for exclusion. Review articles and meta-analyses were screened to obtain additional eligible studies. Search results were then reviewed, and articles were included if the study investigated vacuum-assisted biopsy for benign breast tumor.

The inclusion criteria for the present review were as follows: (1) patients with a benign breast tumor, (2) patients who underwent vacuum-assisted biopsy, (3) study comprised relevant outcomes such as operative and postoperative measurements, and (4) odds ratio (OR) or proportional data reported, or data provided for their calculation. Articles that studied other diseases, non-original articles, or non-English language publications were excluded. Complete resection was defined as no remnant lesion identified on postoperative or postprocedural sonographic examination. Recurrence was defined as a newly occurring lesion within the previously excised region.

\subsection{Data Extraction}

Data from all eligible studies were extracted by two investigators. Extracted data from each of the eligible studies included the following: the first author's name, year of publication, study location, study design, study period, number of patients analyzed, age, number of lesions, indication of vacuum-assisted biopsy, complete resection rate, recurrence rate, and complications.

\subsection{Quality Assessment}

We used the Newcastle-Ottawa quality assessment scale (NOS) to assess the risk of bias in observational studies [8]. NOS uses a star system that includes three domains: selection, comparability, and exposure/outcome. All studies were independently reviewed by two investigators. Any disagreement concerning study selection and data extraction was resolved by consensus.

\subsection{Statistical Analysis}

To obtain the estimated effect sizes, a meta-analysis was performed using the Comprehensive Meta-Analysis software package (Biostat, Englewood, NJ, USA). We computed the point estimate by combining single descriptive statistics to pool the overall proportions [9]. Pooled proportion of event was estimated using fixed-effect and random-effect model of meta-analysis. To pool the proportion (complete resection, recurrence, and complications), we used logit-transformed values to avoid the squeezing of variance effect $[10,11]$ Confidence interval was calculated using the exact confidence limits for a binominal proportion [11]. To pool the odds ratio for binary data, we used the inverse variance method with random effects weighing for meta-analysis of outcomes. As the eligible studies used populations with heterogeneity, a random-effects model was more appropriate than a fixed-effects model. Heterogeneity between eligible studies was checked using Cochran's $\mathrm{Q}$ (Chi-square test) ( $p$-value $<0.10$ were considered significant). Egger's test was conducted to evaluate the publication bias.

We performed a subgroup analysis to assess the heterogeneity across the studies. The pooled incidence of complete resection, recurrence, and complications was calculated according to study-level characteristics as follows: (1) mass removal technique (VAE or 
surgical open excision) (2) breast tumor size. We also conducted a meta-regression test for each moderator to assess heterogeneity.

\section{Results}

\subsection{Selection and Characteristics}

A total of 509 studies were identified through a database search. Among the searched studies, 236 were excluded. Studies were excluded because they were non-original $(n=31)$, studied other diseases $(n=24)$, or were written in a non-English language $(n=39)$. Finally, 26 studies [12-37] comprising 18,170 patients were included in the present meta-analysis (Figure 1), and detailed information about the eligible studies is shown in Table 1.

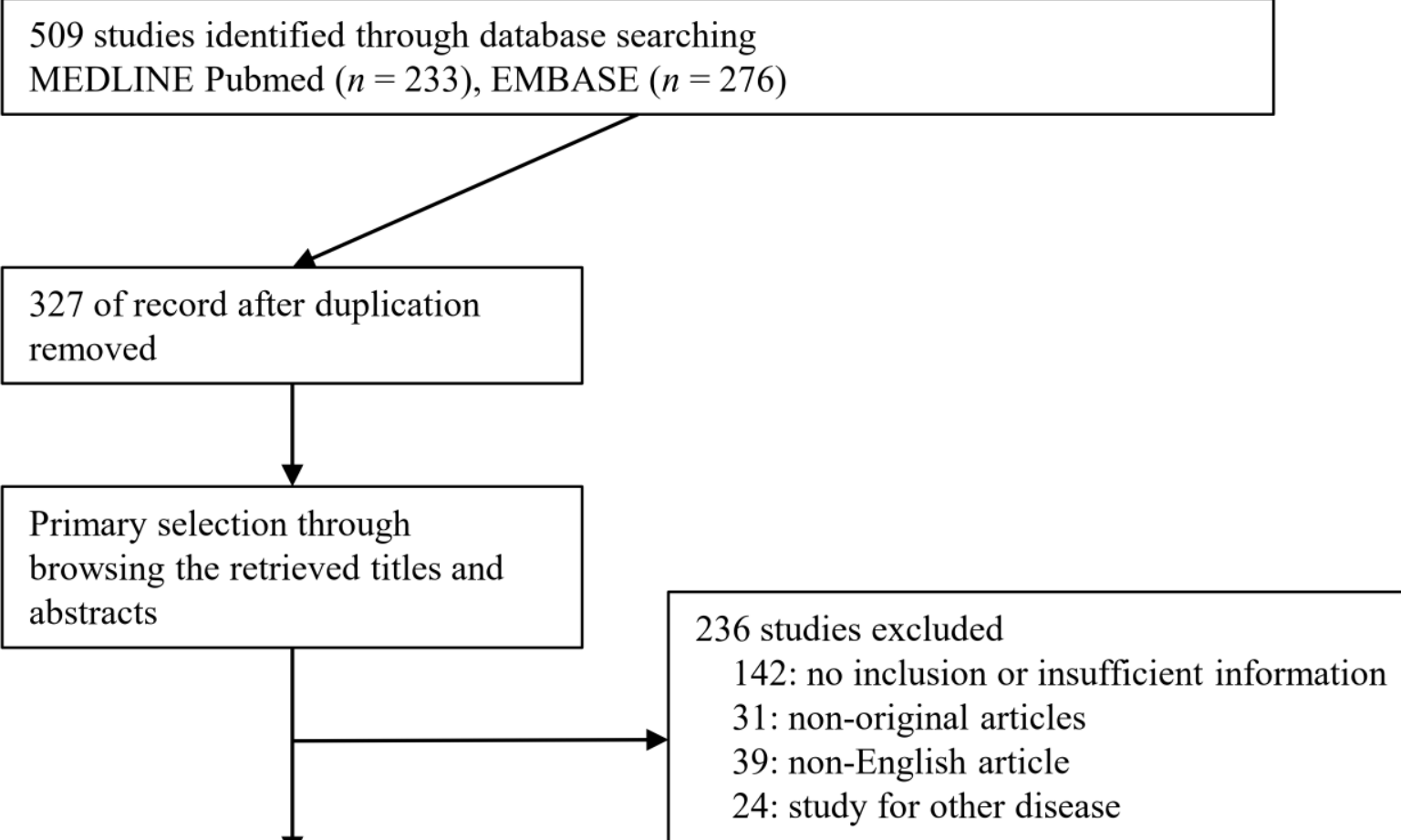

91 full-text articles assessed for eligibility

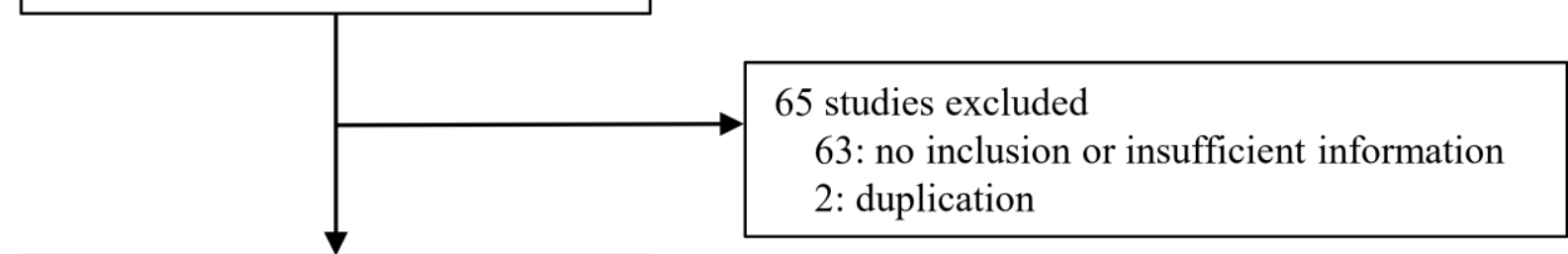

26 studies included in the metaanalysis

Figure 1. Flowchart summarizes literature and study selection. 
Table 1. Main characteristics of the eligible studies.

\begin{tabular}{|c|c|c|c|c|c|c|c|c|c|c|c|}
\hline & Study, Year & Location & $\begin{array}{l}\text { Study } \\
\text { Design }\end{array}$ & $\begin{array}{l}\text { Study } \\
\text { Period }\end{array}$ & $\begin{array}{c}\text { Number } \\
\text { of } \\
\text { Patients }\end{array}$ & $\begin{array}{c}\text { Number } \\
\text { of } \\
\text { Lesions }\end{array}$ & Tx & Dx/Lesion & Indication & Subgroup & Gauge \\
\hline 1 & Fine 2001 [12] & USA, single center & Observational & 1999-1999 & 45 & 50 & VAE & Variable & $\begin{array}{c}\text { Visualized } \\
\text { breast lesion on } \\
\text { mammography } \\
\text { or ultrasonog- } \\
\text { raphy }\end{array}$ & & 11 \\
\hline 2 & $\begin{array}{l}\text { Perez-Fuentes } \\
2001 \text { [13] }\end{array}$ & Venezuela, single center & Observational & 1998-2000 & 83 & 88 & VAE & Variable & $\begin{array}{c}\text { BI-RADS } \\
\text { Category 2-5 }\end{array}$ & & 11 \\
\hline 4 & Fine 2003 [15] & USA, multicenter & Observational & ND & $\begin{array}{l}104 \\
216\end{array}$ & & $\begin{array}{c}\mathrm{OE} \\
\mathrm{VAE}\end{array}$ & $\begin{array}{l}\text { Variable } \\
\text { Variable }\end{array}$ & $\begin{array}{l}\text { Low risk } \\
\text { palpable mass }\end{array}$ & 8 & 8 \\
\hline 5 & $\begin{array}{c}\text { Alonso-Bartolome } \\
2004 \text { [16] }\end{array}$ & Spain, single center & Observational & 2000-2002 & 97 & 102 & VAE & Variable & $\begin{array}{l}\text { BI-RADS } \\
\text { Category } 3\end{array}$ & 11 & $\begin{array}{l}11 \\
11\end{array}$ \\
\hline 6 & $\begin{array}{c}\text { Krainick-Strobel } \\
2007 \text { [17] }\end{array}$ & Germany, single center & Observational & 2000-2003 & 45 & 46 & VAE & Variable & $\begin{array}{l}\text { BI-RADS } \\
\text { Category } 3\end{array}$ & & 8 or 11 \\
\hline 7 & Ko 2008 [18] & Korea, single center & Observational & $2002-2003$ & 199 & 263 & VAE & Variable & $\begin{array}{l}\text { BI-RADS } \\
\text { Category } 3\end{array}$ & & 8 or 11 \\
\hline 8 & Wang 2009 [19] & China, single center & Observational & $2007-2008$ & 244 & & VAE & Variable & $\begin{array}{l}\text { BI-RADS } \\
\text { Category } 3\end{array}$ & Benign & 10 \\
\hline 9 & Wang 2009 [20] & China, single center & Observational & 2004-2006 & 62 & 150 & VAE & Variable & $\begin{array}{c}\text { BI-RADS } \\
\text { Category 1-3 }\end{array}$ & & 8 or 11 \\
\hline 10 & Maxwell 2009 [21] & UK, single center & Observational & $2002-2008$ & $\begin{array}{c}36 \\
1 \\
12 \\
13\end{array}$ & 87 & $\begin{array}{l}\text { OE } \\
\text { VAE }\end{array}$ & $\begin{array}{l}\text { Variable } \\
\text { Papilloma } \\
\text { Papilloma } \\
\text { Papilloma }\end{array}$ & $\begin{array}{l}\text { papilloma } \\
\text { papilloma } \\
\text { papilloma }\end{array}$ & $\begin{array}{c}\text { EnCor } \\
\text { Mammotome } \\
\text { Mammotome }\end{array}$ & $\begin{array}{c}7 \\
8 \\
11\end{array}$ \\
\hline 11 & Yom 2009 [22] & Korea, single center & Observational & 2001-2004 & 150 & & VAE & & $\begin{array}{l}\text { BI-RADS } \\
\text { Category 2-4 }\end{array}$ & Within 2 yr & 8 \\
\hline \multirow{3}{*}{12} & \multirow{3}{*}{ Hahn 2009 [23] } & \multirow{3}{*}{ Germany, single center } & \multirow{3}{*}{ Observational } & \multirow{3}{*}{ 2006-2007 } & 184 & & VAE & & \multirow{3}{*}{$\begin{array}{c}\text { BI-RADS } \\
\text { Category 3-5 }\end{array}$} & After 2 yr & 8 \\
\hline & & & & & & & & Variable & & Overall & \\
\hline & & & & & $\begin{array}{c}21 \\
17 \\
9 \\
15\end{array}$ & & $\begin{array}{l}\text { VAE } \\
\text { VAE } \\
\text { VAE } \\
\text { VAE }\end{array}$ & $\begin{array}{l}\text { Variable } \\
\text { Variable } \\
\text { Variable } \\
\text { Variable }\end{array}$ & & $\begin{array}{l}\text { Mammotome } \\
\text { ATEC } \\
\text { Mammotome } \\
\text { ATEC }\end{array}$ & $\begin{array}{c}8 \\
9 \\
11 \\
12\end{array}$ \\
\hline
\end{tabular}


Table 1. Cont.

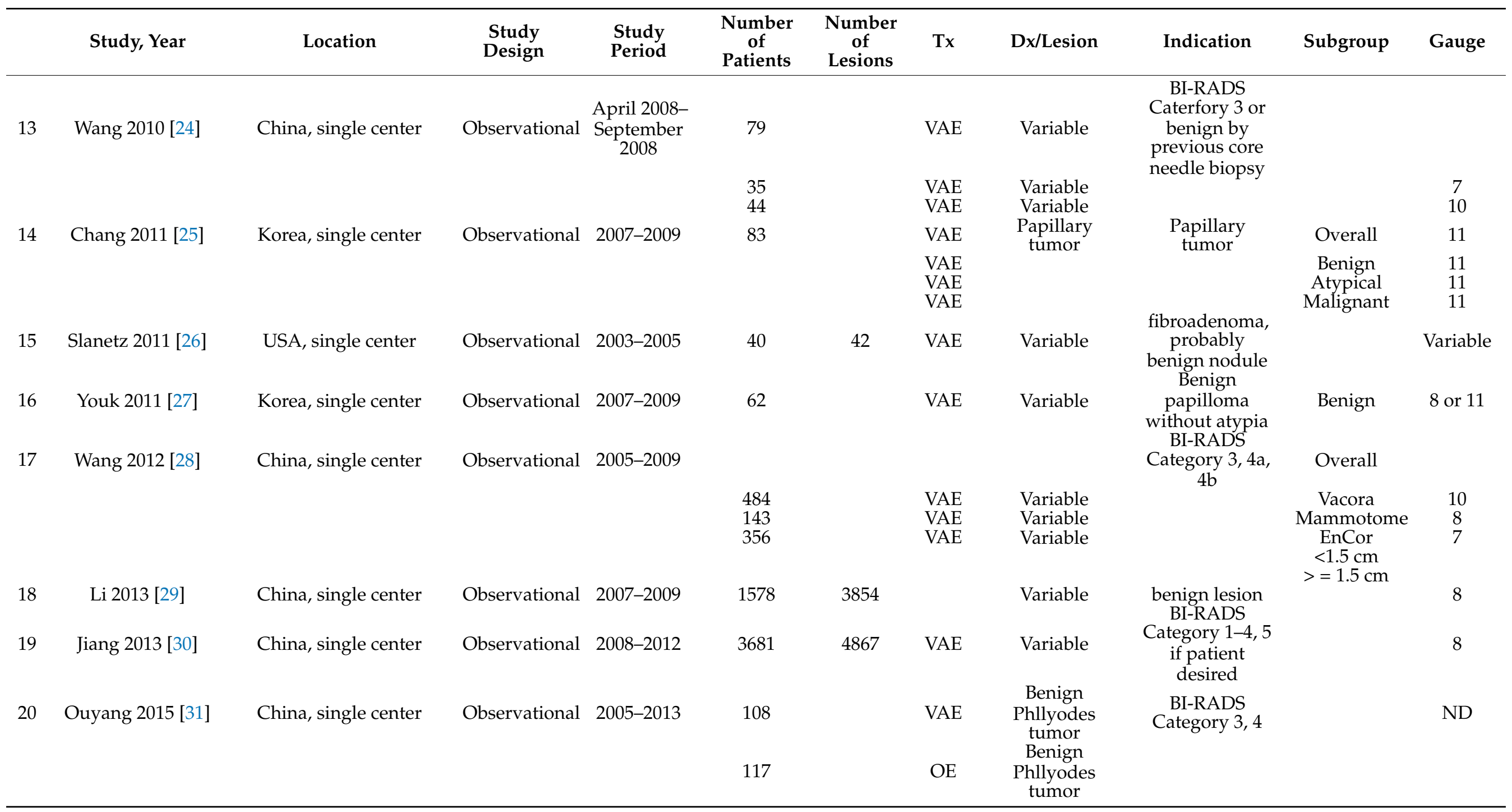


Table 1. Cont.

\begin{tabular}{|c|c|c|c|c|c|c|c|c|c|c|c|}
\hline & Study, Year & Location & $\begin{array}{l}\text { Study } \\
\text { Design }\end{array}$ & $\begin{array}{l}\text { Study } \\
\text { Period }\end{array}$ & $\begin{array}{c}\text { Number } \\
\text { of } \\
\text { Patients }\end{array}$ & $\begin{array}{c}\text { Number } \\
\text { of } \\
\text { Lesions }\end{array}$ & Tx & Dx/Lesion & Indication & Subgroup & Gauge \\
\hline \multirow[t]{2}{*}{21} & Kim 2016 [32] & Korea, single center & Observational & 2002-2012 & 126 & & $\mathrm{OE}$ & $\begin{array}{l}\text { Benign } \\
\text { Phllyodes } \\
\text { tumor } \\
\text { Benign }\end{array}$ & $\begin{array}{c}\text { BI-RADS } \\
\text { Category 3, } 4\end{array}$ & & \\
\hline & & & & & 20 & & VAE & $\begin{array}{l}\text { Phllyodes } \\
\text { tumor }\end{array}$ & & & 11 or 8 \\
\hline \multirow[t]{2}{*}{22} & Bugdayci 2017 [33] & Turkey, single center & Observational & 1999-2001 & 51 & & & Fibroadenoma & $\begin{array}{c}\text { BI-RADS } \\
\text { Category } 3,4 \mathrm{~A}\end{array}$ & Overall & 11 \\
\hline & & & & & $\begin{array}{c}13 \\
32 \\
6\end{array}$ & & & & & $\begin{array}{l}<1 \mathrm{~cm} \\
1-2 \mathrm{~cm} \\
2-3 \mathrm{~cm}\end{array}$ & \\
\hline 23 & Park 2018 [34] & Korea, single center & Observational & 2003-2015 & 8748 & 11,221 & VAE & Variable & $\begin{array}{c}\text { BI-RADS } \\
\text { Category 3,4A }\end{array}$ & & 8 \\
\hline 24 & Salazar 2018 [35] & Spain, single center & Observational & 2012-2016 & 143 & 152 & VAE & & benign lesion & $\begin{array}{l}\text { After tx } \\
\text { FU } 6 \mathrm{mo} \\
<16 \mathrm{~mm} \\
>=16 \mathrm{~mm}\end{array}$ & 10 or 7 \\
\hline 25 & Choi 2019 [36] & Korea, single center & Observational & 2005-2015 & 233 & & VAE & & $\begin{array}{c}\text { Benign } \\
\text { papilloma } \\
\text { without atypia }\end{array}$ & & 11 or 8 \\
\hline \multirow[t]{2}{*}{26} & Wang 2019 [37] & China, single center & Observational & 2008-2016 & $\begin{array}{l}206 \\
101\end{array}$ & & $\begin{array}{c}\mathrm{OE} \\
\mathrm{VAE}\end{array}$ & $\begin{array}{l}\text { Intraductal } \\
\text { papilloma }\end{array}$ & $\begin{array}{c}\text { BI-RADS } \\
\text { Category } 3,4 \mathrm{~A},\end{array}$ & & 7 \\
\hline & & & & & & & & & & $\begin{array}{c}<1 \mathrm{~cm} \\
>=1 \mathrm{~cm}\end{array}$ & \\
\hline
\end{tabular}

Abbreviation; BI-RADS, breast imaging, reporting and data system; VAE, vacuum assisted excisional biopsy; OE, open excision; ND, non-descriptive. 
All were observational studies, and there were no randomized controlled trials (Table 1). Two studies included benign phyllodes tumors [31,32]. The indications of 16 studies vary from BI-RADS 1-4b [11,16-20,22-24,28,30-34,37]. Perez-Fuentes et al. include eight patients with BI-RADS 5 [13] Hahn et al. included two patients with BI-RADS 5 [23] Jiang et al. included 48 patients with BI-RADS 5 in cases where patients desired biopsy [30]. Sixteen studies were conducted in Asian countries [14,18-20,22,24,25,27-32,34,36,37].

\subsection{Quality Assessment}

The quality assessment and risk of bias for each eligible study are summarized in Table 2. All the included studies were observational. The NOS score of 11 studies (42.3\%) was 4 points (lowest) and that of nine studies (34.6\%) was 7 points (highest). We found that all studies had insufficient selection of controls in the selection domain and non-response rate in the exposure domain. Overall, substantial confounding factors may exist with respect to selection and exposure. Particularly, only four studies directly compared VAE with open excision.

\subsection{Complete Resection and Recurrence Rate}

The estimated rates of complete resection and recurrence through vacuum-assisted and open excisions are summarized in Table 3 . The meta-analysis showed that the complete resection rate of VAE was 0.930 (95\% confidence interval [CI], 0.897-0.954; Cochran's Q, $p<0.001$; Egger's test, $p=0.566)[13,15-18,20,22-28,33-37]$. In the meta-regression test, there was no significant difference $(p=0.154)$. The meta-analysis showed a recurrence rate of 0.039 (95\% CI, 0.016-0.091; Cochran's Q, $p<0.001$; Egger's test, $p=0.243$ ) in the VAE group [21,29-32]. The meta-regression test showed no statistical significance $(p=0.896)$. Subgroup analysis according to tumor size is summarized in Figure 2. Three studies comprised subgroups that were divided by $1.0 \mathrm{~cm}$ of tumor size (Figure 2A) [12,33,37]. The estimated rates of complete resection of the subgroup with a tumor size $<1.0 \mathrm{~cm}$ was 0.870 (95\% CI, 0.494-0.979; Cochran's Q, $p=0.002$; Egger's test, $p=0.515$ ), whereas that of the subgroup with a tumor size $>1.0 \mathrm{~cm}$ was 0.760 (95\% CI, 0.672-0.831; Cochran's $Q$, $p=0.359)$. The meta-regression test showed no statistical significance $(p=0.489)$. Two studies comprised subgroups that were divided by $1.5 \mathrm{~cm}$ tumor size (Figure 2B) [28,35]. The estimated rates of complete resection of the subgroup with a tumor size $<1.5 \mathrm{~cm}$ was 0.966 (95\% CI, 0.924-0.986; Cochran's $Q, p=0.163$ ), whereas that of the subgroup with a tumor size $>1.0 \mathrm{~cm}$ was 0.900 (95\% CI, $0.830-0.943$; Cochran's $Q, p=0.060)$. The meta-regression test showed statistical significance $(p=0.021)$. 
Table 2. NOS for the risk of bias and quality assessment of NRSs.

\begin{tabular}{|c|c|c|c|c|c|c|c|c|c|c|}
\hline \multirow[b]{2}{*}{$\begin{array}{l}\text { Study } \\
\text { No. }\end{array}$} & \multirow[b]{2}{*}{ Author, Year } & \multicolumn{4}{|c|}{ Selection } & \multirow{2}{*}{$\begin{array}{c}\text { Comparability } \\
\text { Control for } \\
\text { Important or } \\
\text { Additional } \\
\text { Factors }\end{array}$} & \multicolumn{3}{|c|}{ Exposure } & \multirow[b]{2}{*}{$\begin{array}{l}\text { Total } \\
\text { Score }\end{array}$} \\
\hline & & $\begin{array}{c}\text { Adequate } \\
\text { Definition } \\
\text { of Patient } \\
\text { Cases }\end{array}$ & $\begin{array}{l}\text { Representativeness } \\
\text { of Patient Cases }\end{array}$ & $\begin{array}{l}\text { Selection of } \\
\text { Controls }\end{array}$ & $\begin{array}{l}\text { Definition of } \\
\text { Controls }\end{array}$ & & $\begin{array}{l}\text { Ascertainment } \\
\text { of Exposure }\end{array}$ & $\begin{array}{l}\text { Same Method of } \\
\text { Ascertainment } \\
\text { for Participants }\end{array}$ & $\begin{array}{c}\text { Nonresponse } \\
\text { Rate }\end{array}$ & \\
\hline 1 & Fine 2001 [12] & $\star$ & $\star$ & 夫 & $\star$ & * & $\star$ & * & & 7 \\
\hline 2 & Perez-Fuentes 2001 [13] & $\star$ & $\star$ & $\star$ & & & $\star$ & & & 4 \\
\hline 3 & Chen 2003 [14] & $\star$ & $\star$ & $\star$ & $\star$ & * & $\star$ & 夫 & & 7 \\
\hline 4 & Fine 2003 [15] & $\star$ & $\star$ & $\star$ & & $\star$ & 夫 & & & 5 \\
\hline 5 & $\begin{array}{c}\text { Alonso-Bartolome } \\
2004 \text { [16] }\end{array}$ & $\star$ & $\star$ & & & & $\star$ & $\star$ & & 4 \\
\hline 6 & $\begin{array}{c}\text { Krainick-Strobel } \\
2007 \text { [17] }\end{array}$ & $\star$ & $\star$ & & & & $\star$ & $\star$ & & 4 \\
\hline 7 & Ko 2008 [18] & 夫 & $\star$ & $\star$ & & & $\star$ & $\star$ & & 5 \\
\hline 8 & Wang 2009 [19] & $\star$ & $\star$ & & & & $\star$ & $\star$ & & 4 \\
\hline 9 & Wang 2009 [20] & $\star$ & $\star$ & $\star$ & $\star$ & * & $\star$ & $\star$ & & 7 \\
\hline 10 & Maxwell 2009 [21] & $\star$ & $\star$ & 夫 & & & 夫 & 夫 & & 5 \\
\hline 11 & Yom 2009 [22] & $\star$ & $\star$ & & & & $\star$ & $\star$ & & 4 \\
\hline 12 & Hahn 2009 [23] & $\star$ & $\star$ & $\star$ & & & $\star$ & $\star$ & & 5 \\
\hline 14 & Chang 2011 [25] & * & $\star$ & $\star$ & $\star$ & * & $\star$ & $\star$ & & 7 \\
\hline 15 & Slanetz 2011 [26] & $\star$ & $\star$ & & & & $\star$ & $\star$ & & 4 \\
\hline 16 & Youk 2011 [27] & $\star$ & $\star$ & & & & $\star$ & $\star$ & & 4 \\
\hline 17 & Wang 2012 [28] & $\star$ & $\star$ & & & & $\star$ & $\star$ & & 4 \\
\hline 18 & Li 2013 [29] & * & $\star$ & $\star$ & $\star$ & $\star$ & $\star$ & $\star$ & & 7 \\
\hline 19 & Jiang 2013 [30] & $\star$ & $\star$ & & & & $\star$ & $\star$ & & 4 \\
\hline 20 & Ouyang 2015 [31] & $\star$ & $\star$ & $\star$ & $\star$ & * & $\star$ & $\star$ & & 7 \\
\hline 21 & Kim 2016 [32] & $\star$ & $\star$ & $\star$ & $\star$ & $\star$ & $\star$ & $\star$ & & 7 \\
\hline 22 & Bugdayci 2017 [33] & $\star$ & $\star$ & $\star$ & $\star$ & $\star$ & $\star$ & $\star$ & & 7 \\
\hline 23 & Park 2018 [34] & $\star$ & $\star$ & $\star$ & $\star$ & & $\star$ & $\star$ & & 6 \\
\hline 24 & Salazar 2018 [35] & $\star$ & $\star$ & & & & $\star$ & $\star$ & & 4 \\
\hline 25 & Choi 2019 [36] & $\star$ & $\star$ & $\star$ & $\star$ & 夫 & $\star$ & $\star$ & & 7 \\
\hline 26 & Wang 2019 [37] & $\star$ & $\star$ & & & & 夫 & 夫 & & 4 \\
\hline
\end{tabular}


Table 3. The estimated rates of complete resection and recurrence through vacuum-assisted and open excisions.

\begin{tabular}{|c|c|c|c|c|c|c|}
\hline Subgroup & $\begin{array}{l}\text { Number of } \\
\text { Subsets }\end{array}$ & $\begin{array}{c}\text { Fixed Effect } \\
{[95 \% \text { CI] }}\end{array}$ & $\begin{array}{l}\text { Heterogeneity Test } \\
{[p \text {-Value }]}\end{array}$ & $\begin{array}{l}\text { Random Effect } \\
{[95 \% \text { CI }]}\end{array}$ & $\begin{array}{l}\text { Egger's Test } \\
\text { [p-Value] }\end{array}$ & $\begin{array}{c}\text { Meta-Regression Test } \\
\text { [ } p \text {-Value }]\end{array}$ \\
\hline \multicolumn{7}{|c|}{ Complete resection, rate } \\
\hline $\begin{array}{c}\text { Vacuum-assisted } \\
\text { excision }\end{array}$ & 23 & $0.935[0.931,0.940]$ & $<0.001$ & $0.930[0.897,0.954]$ & 0.566 & 0.154 \\
\hline Open excision & 1 & $0.994[0.907,1.000]$ & 1.000 & $0.994[0.907,1.000]$ & - & \\
\hline \multicolumn{7}{|l|}{ Recurrence, rate } \\
\hline $\begin{array}{l}\text { Vacuum-assisted } \\
\text { excision }\end{array}$ & 5 & $0.018[0.015,0.021]$ & $<0.001$ & $0.039[0.016,0.091]$ & 0.243 & 0.896 \\
\hline
\end{tabular}

CI, Confidence interval. 


\begin{tabular}{|c|c|c|c|c|c|c|}
\hline \multirow{2}{*}{ Group by } & \multirow[t]{2}{*}{ Study name } & \multirow[t]{2}{*}{ Subgroup within study } & \multicolumn{4}{|c|}{ Statistics for each study } \\
\hline & & & $\begin{array}{l}\text { Event } \\
\text { rate }\end{array}$ & $\begin{array}{l}\text { Lower } \\
\text { limit }\end{array}$ & $\begin{array}{l}\text { Upper } \\
\text { limit }\end{array}$ & p-Valt \\
\hline$<1 \mathrm{~cm}$ & Bugdayci 201 & $<1 \mathrm{~cm}$ & 0.538 & 0.282 & 0.776 & 0.782 \\
\hline$<1 \mathrm{~cm}$ & Fine 2001 & $<1 \mathrm{~cm}$ & 0.921 & 0.782 & 0.974 & 0.00 \\
\hline$<1 \mathrm{~cm}$ & Wang 2019 & $<1 \mathrm{~cm}$ & 0.969 & 0.809 & 0.996 & 0.00 \\
\hline$<1 \mathrm{~cm}$ & & & 0.870 & 0.494 & 0.979 & 0.05 \\
\hline$>1 \mathrm{~cm}$ & Bugdayci 201 & $>1 \mathrm{~cm}$ & 0.684 & 0.522 & 0.811 & 0.02 \\
\hline$>1 \mathrm{~cm}$ & Fine 2001 & $>1 \mathrm{~cm}$ & 0.833 & 0.523 & 0.958 & 0.03 \\
\hline$>1 \mathrm{~cm}$ & Wang 2019 & $>1 \mathrm{~cm}$ & 0.797 & 0.686 & 0.876 & 0.000 \\
\hline$>1 \mathrm{~cm}$ & & & 0.760 & 0.672 & 0.831 & 0.00 \\
\hline
\end{tabular}

\section{Event rate and $95 \% \mathrm{Cl}$}
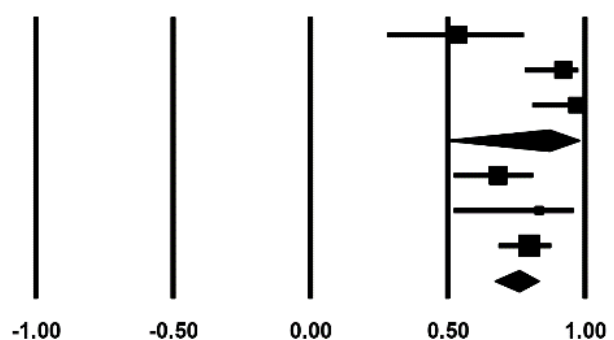

(A)

Group by
Subgroup within study

$<1.5 \mathrm{~cm}$
$<1.5 \mathrm{~cm}$
$<1.5 \mathrm{~cm}$
$>1.5 \mathrm{~cm}$
$>1.5 \mathrm{~cm}$
$>1.5 \mathrm{~cm}$

\begin{tabular}{lllllr} 
Study name Subgroup within study & \multicolumn{5}{c}{ Statistics for each study } \\
& $\begin{array}{c}\text { Event } \\
\text { rate }\end{array}$ & $\begin{array}{c}\text { Lower } \\
\text { limit }\end{array}$ & $\begin{array}{c}\text { Upper } \\
\text { limit }\end{array}$ & p-Value \\
Salazar $2018<1.5 \mathrm{~cm}$ & 0.940 & 0.830 & 0.981 & 0.000 \\
Wang $2012<1.5 \mathrm{~cm}$ & 0.975 & 0.957 & 0.986 & 0.000 \\
& 0.966 & 0.924 & 0.986 & 0.000 \\
Salazar $2018>1.5 \mathrm{~cm}$ & 0.863 & 0.781 & 0.917 & 0.000 \\
Wang $2012>1.5 \mathrm{~cm}$ & 0.922 & 0.894 & 0.942 & 0.000 \\
& 0.900 & 0.830 & 0.943 & 0.000
\end{tabular}

(B)

Figure 2. The estimated rates of complete resection in vacuum-assisted excision based on tumor size. (A) According to $1 \mathrm{~cm}$ of tumor size; (B) According to $1.5 \mathrm{~cm}$ of tumor size.

Figure 3 shows the OR for complete resection according to tumor size [12,28,33]. Smaller tumors $(<1.5 \mathrm{~cm})$ showed a more favorable complete resection rate than those larger than $1.5 \mathrm{~cm}(\mathrm{OR}, 3.162 ; 95 \% \mathrm{CI}, 1.757-5.693 ; p<0.001)$. However, in the criterion of $1.0 \mathrm{~cm}$ of tumor size, there was no significant difference (OR, 1.829; 95\% CI, 0.369-8.983; $p=0.462$ ) between larger and smaller tumors.

\begin{tabular}{|c|c|c|c|c|c|c|}
\hline \multirow{2}{*}{ Group by } & \multirow[t]{2}{*}{$\underline{\text { Study name }}$} & \multirow[t]{2}{*}{ Subgroup within study } & \multicolumn{4}{|c|}{ Statistics for each study } \\
\hline & & & $\begin{array}{l}\text { Odds } \\
\text { ratio }\end{array}$ & $\begin{array}{l}\text { Lower } \\
\text { limit }\end{array}$ & $\begin{array}{l}\text { Upper } \\
\text { limit }\end{array}$ & $\mathrm{p}$-Value \\
\hline $1.0 \mathrm{~cm}$ & Bugdayci 2017 & $1.0 \mathrm{~cm}$ & 0.538 & 0.149 & 1.951 & 0.346 \\
\hline $1.0 \mathrm{~cm}$ & Fine 2001 & $1.0 \mathrm{~cm}$ & 2.333 & 0.341 & 15.952 & 0.388 \\
\hline $1.0 \mathrm{~cm}$ & Wang 2019 & $1.0 \mathrm{~cm}$ & 7.891 & 0.990 & 62.908 & 0.051 \\
\hline $1.0 \mathrm{~cm}$ & & & 1.820 & 0.369 & 8.983 & 0.462 \\
\hline $1.5 \mathrm{~cm}$ & Salazar 2018 & $1.5 \mathrm{~cm}$ & 2.492 & 0.682 & 9.111 & 0.167 \\
\hline $1.5 \mathrm{~cm}$ & Wang 2012 & $1.5 \mathrm{~cm}$ & 3.364 & 1.739 & 6.505 & 0.000 \\
\hline $1.5 \mathrm{~cm}$ & & & 3.162 & 1.757 & 5.693 & 0.000 \\
\hline
\end{tabular}

\section{Odds ratio and $95 \% \mathrm{Cl}$}

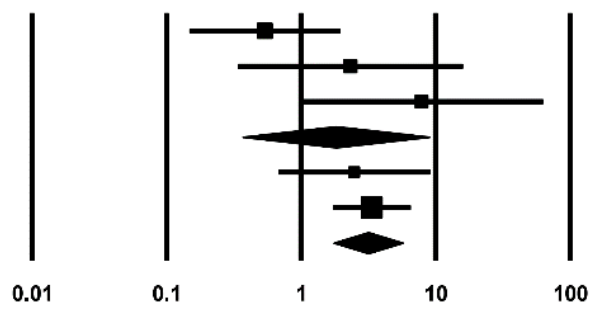

Favors larger tumor Favors smaller tumor

Figure 3. The odds ratio for complete resection in vacuum-assisted excision based on tumor size group.

\subsection{Pooled Incidence of Postoperative Hematoma, Pain, and Ecchymosis}

Postoperative hematomas, pain, and ecchymosis are summarized in Table 4 . The estimated incidence of postoperative hematoma in VAE was 0.092 (95\% CI, 0.067-0.126; Cochran's Q, $p<0.001$; Egger's test 0.668), whereas that after open excision was 0.015 (95\% CI, 0.003-0.073; Cochran's Q, $p=0.305$; meta-regression test, $p=0.049)$ [14-25,28-30,33,35,37]. The estimated incidence of postoperative pain was 0.082 (95\% CI, 0.049-0.134; Cochran's Q, 
$p<0.001$; Egger's test, $p=0.004)[15,16,18,19,21,23-26,28,29,33,35,37]$. The estimated incidence of postoperative ecchymosis was 0.075 (95\% CI, 0.045-0.115; Cochran's $Q, p<0.001$; Egger's test, $p=0.046)[15,16,18-21,23-26,28,29,37]$.

Table 4. The estimated complication rates after vacuum-assisted and open excision.

\begin{tabular}{ccccccc}
\hline Subgroup & $\begin{array}{c}\text { Number } \\
\text { of } \\
\text { Subsets }\end{array}$ & $\begin{array}{c}\text { Fixed Effect } \\
{[95 \% \text { CI }]}\end{array}$ & $\begin{array}{c}\text { Heterogeneity } \\
\text { Test } \\
{[p \text {-Value }]}\end{array}$ & $\begin{array}{c}\text { Random Effect } \\
{[95 \% \text { CI }]}\end{array}$ & $\begin{array}{c}\text { Egger's } \\
\text { Test } \\
{[p \text {-Value }]}\end{array}$ & $\begin{array}{c}\text { Meta- } \\
\text { Regression } \\
\text { Test } \\
{[p \text {-Value }]}\end{array}$ \\
\hline $\begin{array}{c}\text { Hematoma } \\
\text { Vacuum-assisted } \\
\text { excision }\end{array}$ & 25 & $0.088[0.082,0.095]$ & $<0.001$ & $0.092[0.067,0.126]$ & 0.668 & 0.049 \\
$\begin{array}{c}\text { Open excision } \\
\text { Pain }\end{array}$ & 2 & $0.015[0.003,0.073]$ & 0.305 & $0.015[0.003,0.075]$ & - & \\
$\begin{array}{c}\text { Vacuum-assisted } \\
\text { excision }\end{array}$ & 21 & $0.224[0.210,0.240]$ & $<0.001$ & $0.082[0.049,0.134]$ & 0.004 & \\
$\begin{array}{c}\text { Open excision } \\
\text { Ecchymosis }\end{array}$ & 0 & & & & & \\
$\begin{array}{c}\text { Vacuum-assisted } \\
\text { excision }\end{array}$ & 21 & $0.142[0.130,0.156]$ & $<0.001$ & $0.075[0.045,0.115]$ & 0.046 \\
Open excision & 1 & $0.014[0.001,0.182]$ & 1.000 & $0.014[0.001,0.182]$ & - & \\
\hline
\end{tabular}

CI, Confidence interval.

\section{Discussion}

Our analysis suggested that benign breast masses could be safely removed using VAE. The estimated complete resection rate is sufficiently high. Indeed, the estimated rates of recurrence and complications after VAE were sufficiently low. The complete resection and recurrence rate are critical to the use of both diagnostic and therapeutic procedures. However, a limited number of comparative studies and high heterogeneity indicate that more prospective comparative studies are warranted. Nonetheless, our study indicates that VAE is a useful option for successfully removing benign breast masses. However, in terms of high-risk lesions such as phyllodes tumor or atypical ductal hyperplasia, there have been insufficient studies to demonstrate oncologic safety.

VAE was approved by the FDA in 2002 for the removal of benign lesions and by the National Institute for Health and Care Excellence in the United Kingdom in 2006 [5]. However, it is still controversial for some high-risk lesions, such as phyllodes tumors or atypical lesions [5]. To date, several guidelines recommend VAE as an alternative to conventional open excision for benign breast lesions. The international consensus reference in Swiss [6] recommends minimally invasive management of selected B3 lesions with therapeutic VAE. However, according to this consensus, open surgery is still the gold standard for atypical ductal hyperplasia and phyllodes tumors. The study group of breast ultrasonography in Germany [38] stated that VAE allows the resection of breast tissue up to $8 \mathrm{~cm}^{3}$ (volume). However, it has not been formulated as a nationwide guideline. In the statement of the American Society of Breast Surgeons, it was recommended that easily visualized, confirmed histologically prior to treatment, and less than $4 \mathrm{~cm}$ are indications of VAE for fibroadenoma [39]. All these guidelines allow the use of VAE for breast masses that are limited to low-risk lesions.

During our literature search, we found four comparative studies, in which VAE was compared to open excision [14,20,31,32]. All of these studies were observational. Two studies [31,32] comprised benign phyllodes tumors, whereas two [14,20] comprised other benign breast masses such as fibroadenoma, fibrocystic change, papilloma, atypical ductal hyperplasia, or fibrocystic nodule. However, Chen et al. [14] did not report the complete resection rate of all benign masses, in which the complete excision rate of breast carcinomas was reported. Wang et al. [20] reported a 3.4\% incomplete removal rate in the VAE group, which was not significantly different from that of open excision. Two 
studies [31,32] regarding benign phyllodes tumors reported no significant difference in recurrence rate (2.4\% [in VAE] vs. $0 \%$ [in open excision] [32]), (11\% [in VAE] vs. $6.8 \%$ [in open excision] [31]). Complete excision and recurrence rates of VAE were favorable. Although VAE for phyllodes tumors is still controversial [38], our review suggests that the curability of VAE as an alternative to surgery is promising.

Recently, a meta-analysis of 15 studies involving 5256 patients reported the efficacy and safety of VAE [40]. This study showed no significant difference in tumor size, postoperative hematomas, ecchymosis, or residual disease between VAE and open excision. However, the authors used the Chinese database, and many studies are written in the Chinese language. All eligible studies were conducted in China. Only two studies could be searched using the Medline database. This may induce substantial language or location bias [41]. We excluded Chinese-written literature from our analysis. Our study is an updated meta-analysis comprising more recent studies. Another systematic review was conducted without a meta-analysis that aimed to investigate VAE for fibroadenoma [42]. However, this study has some crucial limitations, including the small number of studies $(n=4)$, no assessment of risk of bias, and no pooling estimates.

Our analysis has several limitations. First, all eligible studies were not randomized trials but were observational. There was no prospective study. Selection bias was inevitable. Second, we used single descriptive statistics because there were limited comparative studies. This may have resulted in substantial heterogeneity. To overcome this issue, we conducted subgroup analysis and meta-regression. Third, among the eligible studies, the indication of VAE was substantially heterogeneous, and high-risk or moderate-risk masses were not excluded. Fourth, we included limited a number of studies with open excision because there was a limited number of comparative studies between VAE and open excision. Therefore, the certainty of the comparison of effect size was limited in our meta-analysis. Instead, we noted the single proportional effect size of VAE. Fifth, in Egger's test, publication bias existed regarding two outcomes of pain and ecchymosis. However, other crucial outcomes such as complete resection or recurrence had no publication bias in Egger's Test. In our study, we did not include unpublished studies such as grey literature, dissertation, or conference presentation. In general, studies with significance are more likely to be published [9]. This may contribute to publication bias. Nevertheless, studies that have not been in peer-reviewed journals tend to be of lower quality [9]. We acknowledge that our study may have potential publication bias regarding pain and ecchymosis, but we believe these complications have a less clinical impact than other outcomes. Finally, only articles written in English were included. Overall, our study has a substantial risk of bias due to the nature of a non-randomized study and non-comparative study. The publication bias implies that there might exist more complications. The limitation of our studies emphasizes the need for future study that is comparative with open excision prospectively. Furthermore, it is needed to compare other minimally invasive procedures to remove breast mass. Recently, some procedures such as cryoablation, microwave ablation, highintensity focused ultrasonography, or laser therapy were introduced and some have shown promising results [5]. However, they have still limited evidence and only cryoablation and VAE have received FDA approval [5]. In the future study, systematic review for these procedures would be needed.

\section{Conclusions}

In conclusion, VAE for benign breast lesions showed favorable outcomes with respect to complete resection and complications. This meta-analysis suggested that VAE for low-risk benign breast lesions is a reasonable option for both diagnostic and therapeutic purposes. However, a limited number of comparative studies on open excision have weakened the strength of evidence. More comparative prospective studies are required to estimate the true effect size. 
Author Contributions: Conceptualization: H.-S.Y., W.-S.K., J.-S.P., J.Y. Data curation: H.-S.Y., W.-S.K., J.-S.P., J.Y. Formal analysis: H.-S.Y., W.-S.K., J.-S.P., J.Y. Writing-original: H.-S.Y., W.-S.K., J.-S.P., J.Y. Writing-review \& editing: H.-S.Y., W.-S.K., J.-S.P., J.Y. All authors have read and agreed to the published version of the manuscript.

Funding: This research received no external funding.

Institutional Review Board Statement: Not applicable.

Informed Consent Statement: Not applicable.

Data Availability Statement: Data is contained within the article.

Conflicts of Interest: The authors declare no conflict of interest.

\section{References}

1. Santen, R.J.J.E. Benign Breast Disease in Women. Available online: www.endotext.org (accessed on 2 September 2021).

2. Sisti, A.; Huayllani, M.T.; Boczar, D.; Restrepo, D.J.; Spaulding, A.C.; Emmanuel, G.; Bagaria, S.P.; McLaughlin, S.A.; Parker, A.S.; Forte, A.J. Breast cancer in women: A descriptive analysis of the national cancer database. Acta Bio-Med. Atenei Parm. 2020, 91, 332-341. [CrossRef]

3. Bennett, I.C.; Saboo, A. The Evolving Role of Vacuum Assisted Biopsy of the Breast: A Progression from Fine-Needle Aspiration Biopsy. World J. Surg. 2019, 43, 1054-1061. [CrossRef]

4. Dinç Elibol, F.; Dere, Y.; Belli, A.K.; Elibol, C.; Dere, Ö.; Nazlı, O. Both a biopsy method and a therapeutic procedure in BI-RADS 4A and 4B lesions: Ultrasound-guided vacuum-assisted breast biopsy. Turk. J. Surg. 2020, 36, 65-71. [CrossRef] [PubMed]

5. Roknsharifi, S.; Wattamwar, K.; Fishman, M.D.C.; Ward, R.C.; Ford, K.; Faintuch, S.; Joshi, S.; Dialani, V. Image-guided Microinvasive Percutaneous Treatment of Breast Lesions: Where Do We Stand? Radiographics 2021, 41, 945-966. [CrossRef]

6. Rageth, C.J.; O’Flynn, E.A.M.; Pinker, K.; Kubik-Huch, R.A.; Mundinger, A.; Decker, T.; Tausch, C.; Dammann, F.; Baltzer, P.A.; Fallenberg, E.M.; et al. Second International Consensus Conference on lesions of uncertain malignant potential in the breast (B3 lesions). Breast Cancer Res. Treat. 2019, 174, 279-296. [CrossRef]

7. Moher, D.; Liberati, A.; Tetzlaff, J.; Altman, D.G. Preferred reporting items for systematic reviews and meta-analyses: The PRISMA statement. BMJ 2009, 339, b2535. [CrossRef] [PubMed]

8. Stang, A. Critical evaluation of the Newcastle-Ottawa scale for the assessment of the quality of nonrandomized studies in meta-analyses. Eur. J. Epidemiol. 2010, 25, 603-605. [CrossRef]

9. Borenstein, M.; Hedges, L.V.; Higgins, J.P.; Rothstein, H.R. Introduction to Meta-Analysis; John Wiley \& Sons: Hoboken, NJ, USA, 2009.

10. Schwarzer, G.; Chemaitelly, H.; Abu-Raddad, L.J.; Rücker, G. Seriously misleading results using inverse of Freeman-Tukey double arcsine transformation in meta-analysis of single proportions. Res. Synth. Methods 2019, 10, 476-483. [CrossRef] [PubMed]

11. Barendregt, J.J.; Doi, S.A.; Lee, Y.Y.; Norman, R.E.; Vos, T. Meta-analysis of prevalence. J. Epidemiol. Community Health 2013, 67, 974-978. [CrossRef]

12. Fine, R.E.; Israel, P.Z.; Walker, L.C.; Corgan, K.R.; Greenwald, L.V.; Berenson, J.E.; Boyd, B.A.; Oliver, M.K.; McClure, T.; Elberfeld, J. A prospective study of the removal rate of imaged breast lesions by an 11-gauge vacuum-assisted biopsy probe system. Am. J. Surg. 2001, 182, 335-340. [CrossRef]

13. Perez-Fuentes, J.A.; Longobardi, I.R.; Acosta, V.F.; Marin, C.E.; Liberman, L. Sonographically guided directional vacuum-assisted breast biopsy: Preliminary experience in Venezuela. Am. J. Roentgenol. 2001, 177, 1459-1463. [CrossRef]

14. Chen, S.C.; Yang, H.R.; Hwang, T.L.; Chen, M.F.; Cheung, Y.C.; Hsueh, S. Intraoperative ultrasonographically guided excisional biopsy or vacuum-assisted core needle biopsy for nonpalpable breast lesions. Ann. Surg. 2003, 238, 738-742. [CrossRef] [PubMed]

15. Fine, R.E.; Whitworth, P.W.; Kim, J.A.; Harness, J.K.; Boyd, B.A.; Burak, W.E., Jr. Low-risk palpable breast masses removed using a vacuum-assisted hand-held device. Am. J. Surg. 2003, 186, 362-367. [CrossRef]

16. Alonso-Bartolomé, P.; Vega-Bolívar, A.; Torres-Tabanera, M.; Ortega, E.; Acebal-Blanco, M.; Garijo-Ayensa, F.; Rodrigo, I.; Muñoz-Cacho, P. Sonographically guided 11-G directional vacuum-assisted breast biopsy as an alternative to surgical excision: Utility and cost study in probably benign lesions. Acta Radiol. 2004, 45, 390-396. [CrossRef] [PubMed]

17. Krainick-Strobel, U.; Huber, B.; Majer, I.; Bergmann, A.; Gall, C.; Gruber, I.; Hoffmann, J.; Paepke, S.; Peisker, U.; Walz-Mattmüller, R.; et al. Complete extirpation of benign breast lesions with an ultrasound-guided vacuum biopsy system. Ultrasound Obstet. Gynecol. Off. J. Int. Soc. Ultrasound Obstet. Gynecol. 2007, 29, 342-346. [CrossRef]

18. Ko, E.Y.; Bae, Y.A.; Kim, M.J.; Lee, K.S.; Lee, Y.; Kim, L.S. Factors affecting the efficacy of ultrasound-guided vacuum-assisted percutaneous excision for removal of benign breast lesions. J. Ultrasound Med. Off. J. Am. Inst. Ultrasound Med. 2008, 27, 65-73. [CrossRef]

19. Wang, Z.L.; Li, J.L.; Su, L.; Zhang, Y.F.; Tang, J. An evaluation of a 10-gauge vacuum-assisted system for ultrasound-guided excision of clinically benign breast lesions. Breast 2009, 18, 192-196. [CrossRef] [PubMed]

20. Wang, W.J.; Wang, Q.; Cai, Q.P.; Zhang, J.Q. Ultrasonographically guided vacuum-assisted excision for multiple breast masses: Non-randomized comparison with conventional open excision. J. Surg. Oncol. 2009, 100, 675-680. [CrossRef] 
21. Maxwell, A.J. Ultrasound-guided vacuum-assisted excision of breast papillomas: Review of 6-years experience. Clin. Radiol. 2009, 64, 801-806. [CrossRef] [PubMed]

22. Yom, C.K.; Moon, B.I.; Choe, K.J.; Choi, H.Y.; Park, Y.L. Long-term results after excision of breast mass using a vacuum-assisted biopsy device. ANZ J. Surg. 2009, 79, 794-798. [CrossRef]

23. Hahn, M.; Kagan, K.O.; Siegmann, K.C.; Krainick-Strobel, U.; Kraemer, B.; Fehm, T.; Fischbach, E.; Wallwiener, D.; Gruber, I. Mammotome versus ATEC: A comparison of two breast vacuum biopsy techniques under sonographic guidance. Arch. Gynecol. Obstet. 2010, 281, 287-292. [CrossRef]

24. Wang, Z.L.; Liu, G.; Li, J.L.; Ding, Q.; Su, L.; Tang, J.; Ma, L. Sonographically guided percutaneous excision of clinically benign breast masses. J. Clin. Ultrasound JCU 2011, 39, 1-5. [CrossRef]

25. Chang, J.M.; Han, W.; Moon, W.K.; Cho, N.; Noh, D.Y.; Park, I.A.; Jung, E.J. Papillary lesions initially diagnosed at ultrasoundguided vacuum-assisted breast biopsy: Rate of malignancy based on subsequent surgical excision. Ann. Surg. Oncol. 2011, 18, 2506-2514. [CrossRef] [PubMed]

26. Slanetz, P.J.; Wu, S.P.; Mendel, J.B. Percutaneous excision: A viable alternative to manage benign breast lesions. Can. Assoc. Radiol. J. 2011, 62, 265-271. [CrossRef]

27. Youk, J.H.; Kim, M.J.; Son, E.J.; Kwak, J.Y.; Kim, E.K. US-guided vacuum-assisted percutaneous excision for management of benign papilloma without atypia diagnosed at US-guided 14-gauge core needle biopsy. Ann. Surg. Oncol. 2012, 19, 922-928. [CrossRef] [PubMed]

28. Wang, Z.L.; Liu, G.; Huang, Y.; Wan, W.B.; Li, J.L. Percutaneous excisional biopsy of clinically benign breast lesions with vacuum-assisted system: Comparison of three devices. Eur. J. Radiol. 2012, 81, 725-730. [CrossRef]

29. Li, S.; Wu, J.; Chen, K.; Jia, W.; Jin, L.; Xiao, Q.; Zeng, Y.; Su, F. Clinical outcomes of 1578 Chinese patients with breast benign diseases after ultrasound-guided vacuum-assisted excision: Recurrence and the risk factors. Am. J. Surg. 2013, 205, 39-44. [CrossRef] [PubMed]

30. Jiang, Y.; Lan, H.; Ye, Q.; Jin, K.; Zhu, M.; Hu, X.; Teng, L.; Cao, F.; Lin, X. Mammotome $\left({ }^{\circledR}\right)$ biopsy system for the resection of breast lesions: Clinical experience in two high-volume teaching hospitals. Exp. Ther. Med. 2013, 6, 759-764. [CrossRef] [PubMed]

31. Ouyang, Q.; Li, S.; Tan, C.; Zeng, Y.; Zhu, L.; Song, E.; Chen, K.; Su, F. Benign Phyllodes Tumor of the Breast Diagnosed After Ultrasound-Guided Vacuum-Assisted Biopsy: Surgical Excision or Wait-and-Watch? Ann. Surg. Oncol. 2016, 23, 1129-1134. [CrossRef]

32. Kim, G.R.; Kim, E.K.; Yoon, J.H.; Kim, M.J.; Moon, H.J. Recurrence Rates of Benign Phyllodes Tumors After Surgical Excision and Ultrasonography-Guided Vacuum-Assisted Excision. Ultrasound Q. 2016, 32, 151-156. [CrossRef]

33. Buğdaycı, O.; Kaya, H.; Arıbal, E. Ultrasound Guided Therapeutic Excisional Vacuum Assisted Biopsy in Breast Fibroadenomas. J. Breast Health 2017, 13, 74-76. [CrossRef]

34. Park, H.L.; Kim, K.Y.; Park, J.S.; Shin, J.E.; Kim, H.R.; Yang, B.; Kim, J.Y.; Shim, J.Y.; Shin, E.A.; Noh, S.M. Clinicopathological Analysis of Ultrasound-guided Vacuum-assisted Breast Biopsy for the Diagnosis and Treatment of Breast Disease. Anticancer Res. 2018, 38, 2455-2462. [CrossRef]

35. Salazar, J.P.; Miranda, I.; de Torres, J.; Rus, M.N.; Espinosa-Bravo, M.; Esgueva, A.; Salvador, R.; Rubio, I.T. Percutaneous ultrasound-guided vacuum-assisted excision of benign breast lesions: A learning curve to assess outcomes. Br. J. Radiol. 2019, 92, 20180626. [CrossRef] [PubMed]

36. Choi, H.Y.; Kim, S.M.; Jang, M.; Yun, B.; Kang, E.; Kim, E.K.; Park, S.Y.; Kim, B.; Cho, N.; Moon, W.K. Benign Breast Papilloma without Atypia: Outcomes of Surgical Excision versus US-guided Directional Vacuum-assisted Removal or US Follow-up. Radiology 2019, 293, 72-80. [CrossRef] [PubMed]

37. Wang, Z.L.; Liu, G.; He, Y.; Li, N.; Liu, Y. Ultrasound-guided 7-gauge vacuum-assisted core biopsy: Could it be sufficient for the diagnosis and treatment of intraductal papilloma? Breast J. 2019, 25, 807-812. [CrossRef] [PubMed]

38. Hahn, M.; Krainick-Strobel, U.; Toellner, T.; Gissler, J.; Kluge, S.; Krapfl, E.; Peisker, U.; Duda, V.; Degenhardt, F.; Sinn, H.P.; et al. Interdisciplinary consensus recommendations for the use of vacuum-assisted breast biopsy under sonographic guidance: First update 2012. Ultraschall Med. 2012, 33, 366-371. [CrossRef]

39. Consensus Guideline on the Use of Transcutaneous and Percutaneous Ablation for the Treatment of Benign and Malignant Tumors of the Breast. Available online: www.breastsurgeons.org (accessed on 2 September 2021).

40. Ding, B.; Chen, D.; Li, X.; Zhang, H.; Zhao, Y. Meta analysis of efficacy and safety between Mammotome vacuum-assisted breast biopsy and open excision for benign breast tumor. Gland. Surg. 2013, 2, 69-79. [CrossRef]

41. Higgins, J.P.; Thomas, J.; Chandler, J.; Cumpston, M.; Li, T.; Page, M.J.; Welch, V.A. Cochrane Handbook for Systematic Reviezws of Interventions; John Wiley \& Sons: Hoboken, NJ, USA, 2019.

42. Wahab, T.A.; Szabó, B.K.; Jumah, M.; Elfadl, D.; Behranwala, K.; Chen, I. Efficacy and Safety of Ultrasound Guided VacuumAssisted Excision of Fibroadenomas: A Systematic Review. Open J. Radiol. 2017, 7, 95. [CrossRef] 